Hautarzt 2006 $57: 280$

DOI 10.1007/s00105-006-1120-0

๑) Springer Medizin Verlag 2005

\title{
Functional Food für die Haut
}

Auch der Beitrag von Stahl und Krutmann, in dem es um systemische Photoprotektion durch Carotinoide geht, beschäftigt sich schwerpunktmäßig mit dem Themenkomplex des Wirksamkeitsnachweises von Nahrungsergänzungsmitteln zum Schutz der Haut. Aus dem Beitrag wird ersichtlich, dass die orale Gabe von ausgewählten $\mathrm{Mi}$ kronährstoffen eine sinnvolle und wirksame Komplementärmaßnahme zum externen Sonnenschutz darstellt, die wissenschaftlich fundiert ist. Der Anwender muss sich aber bewusst sein, dass grundlegende Unterschiede hinsichtlich der Wirksamkeitskinetik bestehen: Während zur Entfaltung eines durch Mikronährstoffe bedingten Schutzes der menschlichen Haut vor UV-Strahlung eine Aufbauphase von mehreren Wochen erforderlich ist, ist ein topischer Sonnenschutz natürlich innerhalb von wenigen $\mathrm{Mi}$ nuten bereits effektiv. Andererseits hat die systemische Photoprotektion jedoch auch deutliche Vorteile, da sie dauerhaft und ubiquitär wirkt und somit einen Basisschutz der menschlichen Haut zu leisten vermag.

Der Beitrag von Ekanayake-Mudiyanselage und Thiele beschäftigt sich mit dem Transport von oral gegebenem Vitamin E in die Haut. So konnten neueste Forschungsergebnisse dieser Autoren zeigen, dass die Talgdrüse und ihr Sekret, das Sebum, ein physiologischer Transporter für das fettliebende Vitamin E sind, das so von der Blutzirkulation in die oberen Hautschichten gelangt. Diese Erkenntnisse sind nicht nur für die Thematik „Functinonal Food und Haut" bedeutsam, sondern erweitern auch unser Verständnis der physiologischen Bedeutung des Sebums.

Wir hoffen, dass wir mit diesen Beiträgen eine erste Orientierungshilfe in einem sich zurzeit rasend entwickelnden, hochspannen- den dermatologischen Themengebiet leisten können. Darüber hinausgehende Informationen finden sich in der hier aufgeführten $\mathrm{Mo}$ nographie und Übersichtsarbeit.

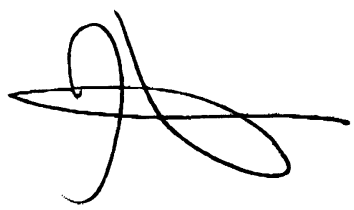

Prof. Dr. J. Krutmann

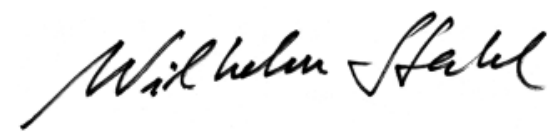

Prof. Dr. W. Stahl

\section{Korrespondierende Autoren \\ Prof. Dr. J. Krutmann}

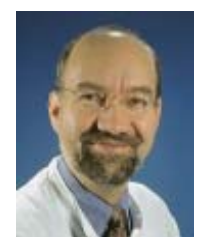

Institut für Umweltmedizinische Forschung, HeinrichHeine-Universität Düsseldorf, Auf'm Hennekamp 50, 40225 Düsseldorf krutmann@rz.uniduesseldorf.de

Prof. Dr. W. Stah

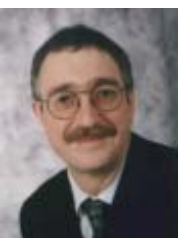

Institut für Biochemie, Heinrich-Heine-Universität Düsseldorf, Postfach 101007, 40001 Düsseldorf wilhelm.stahl@uniduesseldorf.de

\section{Literatur}

1. Gilchrest B, Krutmann J, (eds): Skin Aging. SpringerVerlag, New York, 2006

2. Sies $\mathrm{H}$, Stahl W: Nutritional protection against skin damage from sunlight. Annu Rev Nutr 24: 173200, 2004 